OPEN ACCESS

Edited by:

Ruth Heying,

University Hospital Leuven, Belgium

Reviewed by:

Elumalai Appachi,

Baylor College of Medicine

United States

Lijian Xie,

Shanghai Children's Hospital, China

*Correspondence:

Ho-Chang Kuo

erickuo48@yahoo.com.tw

Specialty section: This article was submitted to

Pediatric Cardiology,

a section of the journal

Frontiers in Pediatrics

Received: 15 May 2019

Accepted: 14 April 2020

Published: 05 May 2020

Citation:

Yan J-H, Chang L-S, Lin Y-J, Guo MM-H, Huang $Y-H$ and Kuo $H-C$

(2020) Clinical Characteristics for

Differentiating Febrile Children With

Suspected Kawasaki Disease

Diagnosis. Front. Pediatr. 8:221

doi: 10.3389/fped.2020.00221

\section{Clinical Characteristics for Differentiating Febrile Children With Suspected Kawasaki Disease Diagnosis}

\author{
Jia-Huei Yan 1,2,3, Ling-Sai Chang ${ }^{1,2}$, Yi-Ju Lin ${ }^{1,2}$, Mindy Ming-Huey Guo ${ }^{1,2}$, \\ Ying-Hsien Huang ${ }^{1,2}$ and Ho-Chang Kuo ${ }^{1,2 *}$
}

${ }^{1}$ Department of Pediatrics, Kaohsiung Chang Gung Memorial Hospital and Chang Gung University College of Medicine, Kaohsiung, Taiwan, ${ }^{2}$ Kawasaki Disease Center, Kaohsiung Chang Gung Memorial Hospital, Kaohsiung, Taiwan,

${ }^{3}$ Department of Pediatrics, Chiayi Chang Gung Memorial Hospital, Chiayi, Taiwan

Background: Kawasaki disease (KD) is a form of vasculitis that primarily affects children under the age of 5 years old. Patients may be missed or diagnosis delayed when initial clinical symptoms do not fulfill the traditional criteria or a normal echocardiography was found. In this study, we aimed to analyze factors that clinicians could use to differentiate febrile children suspected of KD.

Method: We retrospectively enrolled in this study a total of 50 febrile children who were initially suspected of $\mathrm{KD}$, but they did not meet the American Heart Association (AHA) criteria for a diagnosis. However, some of these patients were diagnosed with KD during their second visit. We analyzed patients' characteristics, clinical symptoms, and laboratory data (initial data in the first visit).

Results: In total, 50 patients were enrolled in the study. Of those, ten patients were diagnosed with KD on their second visit (group 1), while the other 40 patients still did not fit a KD diagnosis (group 2). A higher neutrophil-to-lymphocyte ratio (NLR, $p=0.037$ ) and higher $\mathrm{C}$-reactive protein levels (CRP, $p=0.02$ ) were found in group 1 when compared to group 2. A patient with a NLR $>1.33$ combined with a CRP more than $33 \mathrm{mg} / \mathrm{L}$ was more likely to have KD (Sensitivity 90\%, specificity 69.2\%, $p=0.001$; Odds ratio 20.25, 95\% confident interval 2.3-178.25).

Conclusion: Among patients suspected of KD that did not initially meet the criteria, clinicians should pay special attention to elevated neutrophil-to-lymphocyte ratios and CRP levels and closely follow up such patients.

Keywords: clinical characteristics, febrile children, kawasaki disease, C-reactive protein, neutrophil-to-lymphocyte ratio

\section{INTRODUCTION}

Kawasaki disease $(\mathrm{KD})$ is characterized as a medium-sized vasculitis that particularly involves the coronary arteries. It is the most common cause of acquired heart disease in children under the age of 5 years old (1). The etiology of KD is still not completely known. The incidence of KD is higher in Asia than in the United States and Europe and has been increasing in recent decades $(2,3)$. 
In Taiwan and Japan, the incidence is 3 to 15 times higher than in North America (1). KD can lead to coronary artery anomalies (CAA) if not properly treated with intravenous immunoglobulin (IVIG). In the pre-IVIG era, the CAA incidence rate was $20-25 \%$, but IVIG therapy has reduced that to $3-5 \%(1,4)$. Delaying both diagnosis and treatment are major risk factors of CAA persistence and such adverse cardiac events as myocardial ischemia and sudden cardiac arrest (4). The importance of early differentiation of $\mathrm{KD}$ from other fever patients cannot be overlooked.

$\mathrm{KD}$ diagnostic criteria is currently based on clinical findings, echocardiogram, and AHA supplementary laboratory data, so that clinicians can exclude other similar diseases (1). However, $\mathrm{KD}$ diagnosis can often be missed or postponed in the case of atypical or incomplete clinical presentation. The characteristic clinical features of $\mathrm{KD}$ include prolonged fever that lasts more than 5 days, and four of the following five symptoms: oral changes, non-exudative conjunctivitis, skin rash, extremity changes, and cervical lymphadenopathy (1, 5). Nevertheless, these symptoms and signs may appear at different times during the febrile period, and the presentation of illness is often not apparent in infants younger than 6 months old or incomplete KD patients $(1,6,7)$. Pyuria may be treated as a urinary tract infection while subsequent skin rash, injected eyes, or red lips may be considered a reaction to antibiotics. KD may cause retropharyngeal edema and $\mathrm{KD}$ shock syndrome, which are often mistaken as being bacterial in origin (8). In this study, we reviewed suspected KD patients that did not fulfill the criteria at their first clinic visit, comparing and differentiating their clinical findings regarding $\mathrm{KD}$ diagnosis between it and their second visit.

\section{METHOD}

\section{Study Design}

We retrospectively reviewed 50 children who were suspected of $\mathrm{KD}$ by pediatric clinicians, but whose clinical conditions did not meet the American Heart Association's (AHA) criteria for diagnosing typical KD or incomplete KD (1) on the first visit. The cases were divided into two groups based on whether they fit the $\mathrm{KD}$ diagnosis on the second visit. For this study, we examined patients that visited Kaohsiung Chang-Gung Memorial Hospital from November 2008 to September 2018, and patients without complete medical chart records were excluded.

The AHA criteria for typical KD diagnosis is based on fever $\geq 5$ days and the presence of $\geq 4$ of the 5 clinical features (as our rapid memory method 1-2-3-4-5) (5): oral changes (1 mouth) (strawberry tongue, erythematous or cracking of lip, and/or erythema of oral mucosa), non-exudative bilateral bulbar conjunctival injection (2 eyes), cervical lymphadenopathy (3 fingers to check neck lymph node $\geq 1.5 \mathrm{~cm}$ diameter, usually unilateral), 4 limbs extremity changes (erythema and edema of the hands and feet and/or periungual desquamation), and dysmorphism skin rash (5 means many rashes). Incomplete $\mathrm{KD}$ is defined as children with fever $\geq 5$ days, CRP $\geq 30$ $\mathrm{mg} / \mathrm{L}$, and/or ESR $\geq 40 \mathrm{~mm} /$ hour, as well as two or three compatible criteria plus a positive echocardiogram finding or three or more of six laboratory features (anemia by age, platelet count $\geq 450000 / \mathrm{mm}^{3}$ after 7 th day of fever, albumin $\leq 3 \mathrm{~g} / \mathrm{dL}$,
TABLE 1 | Patients' characteristics and clinical symptoms and signs.

KD (Group 1) Not KD (Group 2) P-value

\begin{tabular}{|c|c|c|c|c|}
\hline \multicolumn{2}{|c|}{ Total $(N)$} & 10 & 40 & \\
\hline \multicolumn{2}{|c|}{ Age [year; median (IQR)] } & $2.2(1.5-4.3)$ & $1.4(0.8-3.2)$ & 0.121 \\
\hline \multirow[t]{2}{*}{ Gender } & Male & 5 & 27 & 0.463 \\
\hline & female & 5 & 13 & \\
\hline \multicolumn{2}{|c|}{ Days of fever ${ }^{\star}[$ median $(\mathrm{IQR})]$} & $4.5(3-5)$ & $4(3-6)$ & 0.555 \\
\hline \multicolumn{5}{|c|}{ Initial clinical symptoms and signs [N (\%)] } \\
\hline \multicolumn{2}{|c|}{ Oral change } & $7(70 \%)$ & $25(62.5 \%)$ & 0.73 \\
\hline \multicolumn{2}{|c|}{ Non-exudative conjunctivitis } & $8(80 \%)$ & $26(65 \%)$ & 0.468 \\
\hline \multicolumn{2}{|c|}{ Extremity change } & $4(40 \%)$ & $20(50 \%)$ & 0.728 \\
\hline \multicolumn{2}{|c|}{ Skin rash } & $5(50 \%)$ & $30(75 \%)$ & 0.143 \\
\hline \multicolumn{2}{|c|}{ Lymphadenopathy } & $1(10 \%)$ & 4 (10.3\%) & 1 \\
\hline
\end{tabular}

*Days of fever before first visit to our clinic.

elevated ALT level, WBC count $\geq 15000 / \mathrm{mm}^{3}$, urine $\geq 10$ $\mathrm{WBC} /$ high power field). Based on the AHA recommendations for an incomplete $\mathrm{KD}$ diagnosis, an echocardiogram finding is considered positive if any of the following three conditions are met (1): left anterior descending (LAD) coronary artery or right coronary artery (RCA) $\mathrm{Z}$ score of $\geq 2.5$; presence of a coronary artery aneurysm in the echocardiogram; or the presence of $\geq 3$ other suggestive features, including decreased left ventricular function, mitral regurgitation, pericardial effusion, or $\mathrm{Z}$ scores in LAD coronary artery or RCA of 2 to 2.5. This study was approved the Institutional Review Board of Chang Gung Memorial Hospital (102-0364B).

\section{Data Analysis}

We analyzed the patients' characteristics, clinical symptoms, and laboratory data (initial data of first visit) (Tables 1, 2). Characteristics include patients' age, gender, and days of fever before their first visit. We compared clinical symptoms based on KD diagnostic criteria, including oral changes, non-exudative conjunctivitis, extremity changes, skin rash, and lymphadenopathy $(>1.5 \mathrm{~cm})$. We used the KolmogorovSmirnova test to examine the data and reveal non-normal distributions. We analyzed continuous variables using the Mann-Whitney test, while the chi-square test and Fisher's exact test were adopted for categorical variables. The cut-off point was determined through the ROC curve and Youden index. All data are presented by percentage and median with interquartile range (IQR). We considered $p<0.05$ statistically significant. All statistical tests were performed using SPSS 22.0 (SPSS, Inc., Chicago, Illinois).

\section{RESULTS}

We enrolled 50 patients in this study, including 32 boys and 18 girls, 10 of which were diagnosed as $\mathrm{KD}$ with a positive echocardiogram finding or one or more new clinical features in their second visit (group 1) and 40 non-KD patients (group 2 ). The mean interval between first visit and second visit was 8.4 days. In group 1 , five cases were diagnosed by a 
TABLE 2-1 | Patients' laboratory data [median (IQR)] at first visit.

\begin{tabular}{|c|c|c|c|}
\hline & KD (Group 1) & Not KD (Group 2) & $P$-value \\
\hline Total $(N)$ & 10 & 40 & \\
\hline WBC $\left(1000 / \mathrm{mm}^{3}\right)$ & $11(8.8-13)$ & $11.5(9-15.4)$ & 0.594 \\
\hline Hemoglobulin(g/dL) & $11.3(10.7-12)$ & $11.5(11-12.2)$ & 0.481 \\
\hline Platelet $\left(1000 / \mathrm{mm}^{3}\right)$ & 297.5 (248.5-408.5) & 313.5 (259.5-392.8) & 0.799 \\
\hline Neutrophil (\%) & 63.7 (51.9-77) & $53(43-65)$ & 0.051 \\
\hline Lymphocyte (\%) & $29.7(14-32.7)$ & $37.9(25.2-49.9)$ & $0.048^{*}$ \\
\hline $\begin{array}{l}\text { Neutrophil to } \\
\text { lymphocyte ratio }\end{array}$ & $2.1(1.5-5.5)$ & $1.39(0.85-2.6)$ & $0.037^{\star}$ \\
\hline CRP (mg/L) & 62.3(35.7-99.9) & $26(8.3-48.9)$ & $0.020^{*}$ \\
\hline GOT (IU/L) & $33(24-52)$ & $35.5(28-44)$ & 0.617 \\
\hline GPT (IU/L) & $21(12-55.8)$ & $22(14.8-32)$ & 0.949 \\
\hline Albumin (g/dL) & $4.1(3.8-4.2)$ & $4.2(4-4.4)$ & 0.098 \\
\hline 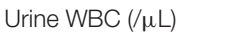 & $6(0-29)$ & $1(0-3)$ & 0.200 \\
\hline
\end{tabular}

${ }^{*} p<0.05$.

TABLE 2-2 | Patients' laboratory data [median (IQR)] at second visit.

\begin{tabular}{|c|c|c|c|}
\hline & KD (Group 1) & Not KD (Group 2) & $P$-value \\
\hline Patient numbers & $10 / 10$ & $7 / 40$ & \\
\hline WBC $\left(1000 / \mathrm{mm}^{3}\right)$ & $11.1(9.6-13.0)$ & $9.6(7.4-11.7)$ & 0.299 \\
\hline Hemoglobulin (g/dL) & $11.0(10.0-11.7)$ & $11.5(10.7-12.2)$ & 0.252 \\
\hline Platelet $\left(1000 / \mathrm{mm}^{3}\right)$ & $544.0(403.5-591.5)$ & $484.0(327.0-527.0)$ & 0.299 \\
\hline Neutrophil (\%) & $55.1(35.5-69.0)$ & $36.8(19.0-56.0)$ & 0.299 \\
\hline Lymphocyte (\%) & $32.0(20.8-59.0)$ & $53.8(25.0-66.0)$ & 0.470 \\
\hline $\begin{array}{l}\text { Neutrophil to lymphocyte } \\
\text { ratio }\end{array}$ & $1.67(0.59-3.55)$ & $0.68(0.29-2.32)$ & 0.408 \\
\hline CRP (mg/L) & $10.3(2.5-26.4)$ & $1.1(0.2-10.1)$ & 0.070 \\
\hline GOT (IU/L) & $33.0(26.8-42.3)$ & $32.0(25.0-50.0)$ & 0.887 \\
\hline GPT (IU/L) & $14.0(8.5-42.0)$ & $21.0(15.0-52.0)$ & 0.417 \\
\hline Albumin (g/dL) & $3.9(3.8-4.4)$ & १ & \\
\hline Urine WBC (/ $\mu \mathrm{L})$ & $0(0-0)$ & ๆી & \\
\hline
\end{tabular}

9/ only 2 patients in group 2 obtained albumin (3.7, and $4.0 \mathrm{~g} / \mathrm{uL}$, respectively) in not KD group in second visit.

919 only 1 patient obtained U-WBC data (61/uL) in not-KD group in second visit. ${ }^{*} p<0.05$.

positive echocardiogram, four cases were diagnosed by new clinical features, and one case was diagnosed by both a positive echocardiogram and new features in the second visit (Figure 1); Of the 10 patients, 6 of them were atypical KD on the diagnosis in second visit. Moreover, in group 1, nine of them had normal echocardiography result and 1 did not perform echocardiography during 1st visit. However, 6 of them had coronary arteries dilatation or aneurysms formation at second visit. The mean ages were $2.2(1.5-4.3)$ years and $1.4(0.8-3.2)$ years old, and the days of fever before the first visit were 4.5 (3-5) days and 4 (3-6) days for group 1 and group 2, respectively. We found no statistically significant differences regarding age, gender, days of fever, and initial clinical symptoms between the two groups (Table 1).

We further analyzed laboratory data and found a higher neutrophil-to-lymphocyte ratio (NLR, $p=0.037$ ) and higher C-reactive protein levels (CRP, $p=0.02)$ in group 1 compared to group 2 (Table 2). Cut-off points determined by ROC curve presented NLR $>1.33$ (AUC $=0.715$, sensitivity $100 \%$, specificity $47.5 \%, p=0.008$, Odds ratio $1.48,95 \%$ confident interval 1.16-1.88) and CRP $>33 \mathrm{mg} / \mathrm{L}(\mathrm{AUC}=0.74$, sensitivity $90 \%$, specificity 59\%, $p=0.011$, Odds ratio $12.94,95 \%$ confident interval 1.49-112.44), both of which have a probability of predicting KD. By combining NLR > 1.33 and CRP > 33 $\mathrm{mg} / \mathrm{L}$, we found a higher odds ratio of 20.25 (95\% confident interval 2.30-178.25) of KD predicting probability, as well as $90 \%$ sensitivity and $69.2 \%$ specificity $(P=0.001)$ (Table 3 ).

\section{DISCUSSION}

In this study, we demonstrated patients who were suspected of $\mathrm{KD}$ but did not meet either AHA diagnostic criteria. A diagnosis of $\mathrm{KD}$ consists of refractory and prolonged fever for more than 5 days and traditional four of five clinical presentation symptoms. However, patients with atypical or incomplete $\mathrm{KD}$ pose a challenge for pediatricians, particularly young infants $<6$ months old and older children at high risk of CAA development $(1,9)$. Atypical or incomplete presentation refers to patients with prolonged fever that only fit two or three clinical features. Symptoms may be dispersed over a period of time; for example, desquamation of the fingers and toes is a late finding that appears at $2 \sim 3$ weeks after onset of fever (10). KD may be misdiagnosed without careful clinical observation or echocardiogram finding. The current treatment recommendation is administering IVIG therapy within 10 days of the onset of illness $(1,11)$, and postponed treatment is the leading cause of CAA formation $(4,7,9-11)$. Clinicians should closely follow up patients who are suspected of KD but do not fit the criteria.

The neutrophil-to-lymphocyte ratio (NLR) is considered the absolute neutrophil count divided by the absolute lymphocyte count and is a simple and inexpensive test to perform. Neutrophils reflect ongoing inflammation and enhanced inflammatory mediator secretion; on the other hand, lymphocytes represent immune regulatory response (12). NLR is a vital biomarker of the balance between inflammation and immune regulation and has been studied with regard to the prognostic and risk factors of cardiovascular diseases and cancer (13-15). Furthermore, previous studies have also demonstrated its predictive value for KD. A higher NLR is associated with IVIG-resistant KD and CAA formation (16-19). Our study demonstrated that the cut-off value of NLR of 1.33 has an odds ratio of 1.48 to predict KD with high sensitivity. Such higher sensitivity can indicate that clinicians should follow up with patients more closely and help them make a real KD diagnosis earlier.

CRP levels are responsible for a patient's inflammation status and can serve as a differentiating factor in this study. According to AHA guidelines for incomplete $\mathrm{KD}$ diagnosis, $\mathrm{CRP}>3.0$ $\mathrm{mg} / \mathrm{dL}(=30 \mathrm{mg} / \mathrm{L})$ and/or ESR $\geq 40 \mathrm{~mm} /$ hour is considered supplementary laboratory data (1). We determined that the CRP cut-off point of more than $33 \mathrm{mg} / \mathrm{L}$ can also be a predicting factor of $\mathrm{KD}$, with an odds ratio of 12.94 (95\% confident interval 1.49112.44 , sensitivity $90 \%$, specificity $59 \%, p=0.011$ ). However, CRP levels are elevated not only in the case of inflammation but also in patients with an infectious disease. Clinicians must cautiously interpret CRP levels and rule out the possibility of 
6

5

4

3

2

1

0

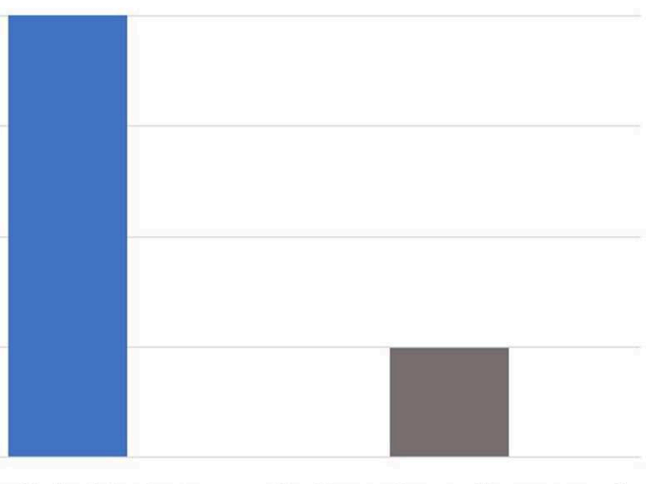

Postive echocardiogram and new feature

FIGURE 1 | Factors for diagnosis of KD based on the second visit.

TABLE 3 | The sensitivity, specificity and odds ratio of cut-points.

\begin{tabular}{|c|c|c|c|}
\hline & NLR $>1.33$ & $\mathrm{CRP}>33 \mathrm{mg} / \mathrm{dL}$ & $\begin{array}{l}\mathrm{NLR}>1.33 \text { plus } \\
\mathrm{CRP}>33 \mathrm{mg} / \mathrm{dL}\end{array}$ \\
\hline Sensitivity & $100 \%$ & $90 \%$ & $90 \%$ \\
\hline Specificity & $47.5 \%$ & $59 \%$ & $69.2 \%$ \\
\hline$P$-value & 0.008 & 0.011 & 0.001 \\
\hline $\begin{array}{l}\text { Odds ratio (95\% } \\
\text { confident interval) }\end{array}$ & $1.48(1.16-1.88)$ & $12.94(1.49-112.44)$ & $\begin{array}{c}20.25 \\
(2.30-178.25)\end{array}$ \\
\hline
\end{tabular}

pathogen invasion and other systemic inflammation diseases, while a CRP level $<33 \mathrm{mg} / \mathrm{L}$ is common in refractory febrile children with infection. Therefore, we combined the two cut-off values of both NLR and CRP to achieve a higher odds ratio of 20.25, a mildly lower sensitivity but better specificity than using NLR or CRP independently (95\% confident interval 2.30-178.25, sensitivity $90 \%$ and specificity $69.2 \%, P=0.001$ ). For patients suspected of KD but that do not meet either of the AHA criteria for typical or incomplete $\mathrm{KD}$, pediatric clinicians should pay particular attention to elevated NLR and CRP levels and closely follow up with those patients. As mentioned in the Table 1, the febrile days before 1st visit ranged from 3 to 6 days. It meant that we should pay attention and check NLR and CRP when we raise the suspicion to KD as early as fever longer than 3 days. A high sensitivity and odds ratio can provide clinicians with a useful tool for differentiation.

This study has certain limitations. First, this study was a retrospectively reviewed study, and the symptoms and onset of febrile days may have had recall bias from patients and family; furthermore, some medical records did not present complete data. Second, the number of patients diagnosed with KD on the second visit was small, which may weaken the study's statistical power, and we hope to obtain more cases to conduct a larger study in the future. Third, this study has a single center, so our results should be examined in another hospital or managed as a cohort study in the future. Further research is still needed about the clinical factors that can distinguish $\mathrm{KD}$ from other febrile diseases.

\section{CONCLUSION}

Identifying atypical presentations of $\mathrm{KD}$ in a timely manner poses a challenge for pediatricians. Delaying treatment can result in cardiovascular sequalae. NLR and CRP are both biomarkers that represent inflammation and immune regulatory pathways and can be used as predictive tools of KD. Among patients suspected of KD that did not initially meet the criteria, clinicians should be aware of an elevated neutrophil-to-lymphocyte ratio, as well as CRP levels, and follow up those patients closely.

\section{DATA AVAILABILITY STATEMENT}

The datasets generated and analyzed during the current study are not publicly available due to strict ethical regulation of information privacy in Taiwan. Requests to access these datasets should be directed to Dr. Ho-Chang Kuo, erickuo48@yahoo.com.tw.

\section{ETHICS STATEMENT}

We retrospectively reviewed those cases' medical records, so we did not obtain the informed consent. On the other hand, our 
study was approved by Institutional Review Board of Chang Gung Memorial Hospital (102-0364B).

\section{AUTHOR CONTRIBUTIONS}

All authors were involved in drafting the article or revising it critically and all authors approved the final version to be published. MG, Y-HH, and $\mathrm{H}-\mathrm{CK}$ contributed to conceptualization and study design of the study, J-HY and Y-JL interpreted and statistically analyzed the data, J-HY was the major contributor in writing the manuscript. L-SC and H-CK were responsible for monitoring and data management, reviewing and editing the manuscript.

\section{REFERENCES}

1. McCrindle BW, Rowley AH, Newburger JW, Burns JC, Bolger AF, Gewitz $M$, et al. Diagnosis, treatment, and long-term management of kawasaki disease: a scientific statement for health professionals from the American Heart Association. Circulation. (2017) 135:e927-99. doi: 10.1161/CIR.0000000000000484

2. Lin MT, Wu MH. The global epidemiology of Kawasaki disease: review and future perspectives. Glob Cardiol Sci Pract. (2017) 2017:e201720. doi: 10.21542/gcsp.2017.20

3. Uehara R, Belay ED. Epidemiology of Kawasaki disease in Asia, Europe, and the United States. J Epidemiol. (2012) 22:79-85. doi: 10.2188/jea.JE20110131

4. Friedman KG, Gauvreau K, Hamaoka-Okamoto A, Tang A, Berry E, Tremoulet $\mathrm{AH}$, et al. Coronary artery aneurysms in kawasaki disease: risk factors for progressive disease and adverse cardiac events in the US Population. J Am Heart Assoc. (2016) 5:e003289. doi: 10.1161/JAHA.116.003289

5. Kuo HC. Preventing coronary artery lesions in Kawasaki disease. Biomed J. (2017) 40:141-6. doi: 10.1016/j.bj.2017.04.002

6. Anderson MS, Todd JK, Glode MP. Delayed diagnosis of Kawasaki syndrome: an analysis of the problem. Pediatrics. (2005) 115:e42833. doi: 10.1542/peds.2004-1824

7. Minich LL, Sleeper LA, Atz AM, McCrindle BW, Lu M, Colan SD, et al. Delayed diagnosis of Kawasaki disease: what are the risk factors? Pediatrics. (2007) 120:e1434-40. doi: 10.1542/peds.2007-0815

8. Vervoort D, Donne M, Van Gysel D. Pitfalls in the diagnosis and management of Kawasaki disease: an update for the pediatric dermatologist. Pediatr Dermatol. (2018) 35:743-7. doi: 10.1111/pde.13620

9. Son MBF, Newburger JW. Kawasaki Disease. Pediatr Rev. (2013) 34:15162. doi: 10.1542/pir.34-4-151

10. Zhu FH, Ang JY. The clinical diagnosis and management of Kawasaki disease: a review and update. Curr Infect Dis Rep. (2016) 18:32. doi: 10.1007/s11908-016-0538-5

11. Bal AK, Prasad D, Umali Pamintuan MA, Mammen-Prasad E, Petrova A. Timing of intravenous immunoglobulin treatment and risk of coronary artery abnormalities in children with Kawasaki disease. Pediatr Neonatol. (2014) 55:387-92. doi: 10.1016/j.pedneo.2013.11.007

12. Azab B, Zaher M, Weiserbs KF, Torbey E, Lacossiere K, Gaddam S, et al. Usefulness of neutrophil to lymphocyte ratio in predicting short- and

\section{FUNDING}

This study was partly supported by grants from the Ministry of Science and Technology, Taiwan (MOST: 105-2314-B-182-050-MY3) and Chang Gung Memorial Hospital (CMRPG8E0211, CMRPG8E0212, and CORPG8F00112 ). These organizations had no role in the study design, data collection and analysis, decision to publish, or preparation of this manuscript.

\section{ACKNOWLEDGMENTS}

We would like to thank the Biostatistics Center, Kaohsiung Chang Gung Memorial Hospital for its statistical work.

long-term mortality after non-ST-elevation myocardial infarction. Am J Cardiol. (2010) 106:470-6. doi: 10.1016/j.amjcard.2010.03.062

13. Bhat T, Teli S, Rijal J, Bhat H, Raza M, Khoueiry G, et al. Neutrophil to lymphocyte ratio and cardiovascular diseases: a review. Expert Rev Cardiovasc Ther. (2013) 11:55-9. doi: 10.1586/erc.12.159

14. Faria SS, Fernandes PC, Jr, Silva MJ, Lima VC, Fontes W, et al. The neutrophil-to-lymphocyte ratio: a narrative review. Ecancermedicalscience. (2016) 10:702. doi: 10.3332/ecancer.2016.702

15. Guthrie GJ, Charles KA, Roxburgh CS, Horgan PG, McMillan DC, Clarke SJ. The systemic inflammation-based neutrophil-lymphocyte ratio: experience in patients with cancer. Crit Rev Oncol Hematol. (2013) 88:21830. doi: 10.1016/j.critrevonc.2013.03.010

16. Cho HJ, Bak SY, Kim SY, Yoo R, Baek HS, Yang S, et al. High neutrophil: lymphocyte ratio is associated with refractory Kawasaki disease. Pediatr Int. (2017) 59:669-74. doi: 10.1111/ped.13240

17. Demir F, Karadeniz C, Ozdemir R, Yozgat Y, Celegen K, Karaaslan U, et al. Usefulness of neutrophil to lymphocyte ratio in prediction of coronary artery lesions in patients with Kawasaki Disease. Balkan Med J. (2015) 32:3716. doi: 10.5152/balkanmedj.2015.151108

18. Ha KS, Lee J, Jang GY, Lee J, Lee KC, Son CS, et al. Value of neutrophillymphocyte ratio in predicting outcomes in Kawasaki disease. Am J Cardiol. (2015) 116:301-6. doi: 10.1016/j.amjcard.2015.04.021

19. Takeshita S, Kanai T, Kawamura Y, Yoshida Y, Nonoyama S. A comparison of the predictive validity of the combination of the neutrophil-to-lymphocyte ratio and platelet-to-lymphocyte ratio and other risk scoring systems for intravenous immunoglobulin (ivig)-resistance in Kawasaki disease. PLoS ONE. (2017) 12:e0176957. doi: 10.1371/journal.pone.0176957

Conflict of Interest: The authors declare that the research was conducted in the absence of any commercial or financial relationships that could be construed as a potential conflict of interest.

Copyright (c) 2020 Yan, Chang, Lin, Guo, Huang and Kuo. This is an open-access article distributed under the terms of the Creative Commons Attribution License (CC $B Y)$. The use, distribution or reproduction in other forums is permitted, provided the original author(s) and the copyright owner(s) are credited and that the original publication in this journal is cited, in accordance with accepted academic practice. No use, distribution or reproduction is permitted which does not comply with these terms. 